\title{
Research on Flight Cyber-Physical System
}

\author{
Jiawei Zheng ${ }^{\text {a) }}$, Lichen Zhang \\ School of Computer, Guangdong University of Technology, Guangdong 510006, China. \\ a) Corresponding author: 1925584561@qq.com
}

\begin{abstract}
As an important application in the field of aviation, the flight Cyber-Physical System (CPS) can play a significant role in the safety and efficiency of the aviation industry. This article focuses on the six characteristics of CPS. Then the dynamic equation of the aircraft in the physical world is established and the state of the aircraft at any moment can be solved. Finally, the functions of the basic flight system are described. With in-depth research, it is possible to analyze flight safety and dispatch.
\end{abstract}

Key words: Cyber-Physical; Flight Physics Modeling.

\section{INTRODUCTION}

The flight Cyber-Physical System (CPS) is the application of CPS in the field of aviation. On the basis of the electronic flight system, the system is more informatization and networked, and it is the development direction of the next-generation flight system. Taking the newly-developed 'dream plane' Boeing 787 passenger aircraft in the United States as an example, it adopts a more advanced sensing technology, with computing power, more robust, intelligent communication and control capabilities, with the ability to connect networks, and more integrated Airborne equipment and software modules. In a global system of sky, satellites, and terrestrial systems, such aircraft are like a single node in flight. They can ensure their own flight safety and can obtain accurate location and time information and can also use this information. Passed in real time to researchers for scientific decision making.

\section{The Features of CPS}

As a comprehensive technology system supporting deep integration of informatization and industrialization, CPS builds an intelligent system that can connect physical space and information space and drives data to flow automatically and realize optimal resource allocation [1]. In the organic operation of the system, functions are assigned to the physical entities in the physical space through the automatic flow of data, and the optimization of specific target resources is realized while exhibiting five typical characteristics [2].

1) Data Driven: Data is the soul of CPS. Data is accumulated in automatic generation, automatic transmission, automatic analysis, automatic execution, and continuous iterative optimization. Fusion can be caused by qualitative change, and resources for the external environment can be optimized.

2) Software definition: Industrial software is the code of all kinds of industrial production rules, supporting most of the manufacturing processes. As a manufacturing-oriented CPS, software has become one of the core vehicles for implementing CPS functions.

3) Virtual mapping: CPS can realize the interaction and linkage between information virtual entities and physical entities to form digital mappings with the ability to perceive, analyze, make decisions, and execute. Optimize configuration efficiency.

4) Heterogeneous integration: CPS can integrate heterogeneous hardware, heterogeneous software, heterogeneous data, and heterogeneous networks to implement data. The automatic flow of different links in the information space and physical space realizes the deep integration of information technology and industrial technology. 
5) System autonomy: CPS can process and analyze in the information space according to the perceived environmental change information and respond to external changes adaptively.

\section{The Description of the Aircraft's Physical Information}

The aircraft consists of a variety of components, including a large number of sensors and actuators. The entire mission of the flight is the highly integrated collaboration of these sensors and actuators and the information network. [3]. Before the physical modeling of the aircraft CPS, we must first determine the ground coordinate system, the aircraft coordinate system, the velocity coordinate system, and define the commonly used kinematic equations.

1) Ground coordinate system: The ground coordinate system $O_{e} X_{e} Y_{e} Z_{e}$ is closely related to the Earth. The origin $O_{e}$ is taken at a certain point on the ground (usually the aircraft's takeoff point is located at the origin). The $O_{e} X_{e}$ axis lies in the ground plane and points in the direction of the flight path. The $O_{e} Z_{e}$ axis goes down vertically and points to the center of the earth. The $O_{e} Y_{e}$ axis is perpendicular to the $X_{e}-O_{e}-Z_{e}$ plane. The ground coordinate system is mainly used to measure the real-time position of the tracking aircraft.

2) Body coordinate system: The body coordinate system $O_{b} X_{b} Y_{b} Z_{b}$, origin $O_{b}$ is taken at the center of gravity of the aircraft. The body coordinate system is fixed with the aircraft. The $O_{b} X_{b}$ axis is located in the plane of symmetry of the aircraft, in line with the axis of the fuselage, and its positive direction is the nose direction. The $O_{b} Z_{b}$ axis is also located in the plane of symmetry, perpendicular to the $O_{b} X_{b}$ axis, with its positive direction pointing below the fuselage. The $O_{b} Y_{b}$ axis is perpendicular to the $X_{b}-O_{b}-Z_{b}$ plane, with its positive direction pointing to the right of the body. The body coordinate system can be used to analyze the force of the aircraft.

3) Velocity Coordinate System: The velocity coordinate system $O_{s} X_{S} Y_{s} Z_{s}$ is taken from the origin of the aircraft and the $O_{s} X_{s}$ axis coincides with the aircraft's flight velocity vector $\mathrm{V}$. The $O_{s} Z_{s}$ axis is within the plane of symmetry of the aircraft and is perpendicular to the $O_{s} X_{s}$ axis. Its positive direction points to the bottom of the fuselage, $O_{s} Y_{s}$ The axis is perpendicular to the $X_{S}-O_{s}-Y_{s}$ plane, with its positive direction pointing to the right of the body.

When the aircraft is flying in the air, the aerodynamic forces Fa on the aircraft include lift $F_{a z}$, resistance Fax, and lateral force Fay. The aerodynamically generated moment Ma includes the yaw moment $M_{a z}$, the roll moment May, and the pitch moment Max. Aerodynamic forces and moments are expressed in the body coordinate system as follows:

$$
\left\{\begin{array}{l}
F_{a z}=\frac{1}{2} C_{-\rho} \rho V^{2} S \\
F_{a y}=\frac{1}{2} C_{j} \rho V^{2} S \\
F_{a z}=\frac{1}{2} C_{z} \rho V^{2} S
\end{array}\right\}
$$

$$
\left\{\begin{array}{l}
M_{a x}=\frac{1}{2} C_{l} \rho V^{2} S b \\
M_{a y}=\frac{1}{2} C_{m} \rho V^{2} S c \\
M_{c c}=\frac{1}{2} C_{n} \rho V^{2} S b
\end{array}\right\}
$$

Where $C_{x}$ is the drag coefficient, $\mathrm{Cy}$ is the lateral force coefficient, $C_{z}$ is the lift coefficient, $\mathrm{Cl}$ is the roll moment coefficient, $\mathrm{Cm}$ is the pitching moment coefficient, $\mathrm{Cn}$ is the yaw moment coefficient, $\rho$ is the air density, $\mathrm{V}$ is the speed of the airplane, $\mathrm{S}$ is the machine Wing effective area, $\mathrm{b}$ is wingspan, $\mathrm{c}$ is draw air string length

The aircraft dynamics equation is established on the basis of Newton's mechanical equations. The linear motion equation of the aircraft under external forces and the rotational equation under external moment are as follows:

$$
\begin{aligned}
& \bar{F}=\frac{d}{d t}(m \bar{V})=m\left(\frac{\partial \bar{V}}{\partial t}+\bar{\Omega} \times \bar{V}\right) \\
& \bar{M}=\frac{d \bar{L}}{d t}=\frac{\partial(\bar{I} \times \bar{V})}{\partial t}+\bar{\Omega} \times(\bar{I} \cdot \bar{\Omega})
\end{aligned}
$$

$\vec{F}$ is the total external force, $\vec{F}=\left(F_{x}, F_{y}, F_{z}\right)^{T} ; \vec{V}$ is the speed vector of the aircraft, $\vec{V}=(u, v, w)^{T} ; \vec{M}$ is the total combined moment, $\vec{M}=\left(M_{x}, M_{y}, M_{z}\right)^{T} ; \vec{L}$ is the momentum moment of the aircraft, $\vec{L}=\left(L_{x}, L_{y}, L_{z}\right)^{T}$; m is the 
quality of the aircraft; $\vec{\Omega}$ is the total angular velocity vector of the dynamic coordinate system relative to the inertial frame, $\vec{\Omega}=(p, q, r)^{T} ; \vec{I}$ is the inertia of the aircraft, $\vec{I}=\left(\begin{array}{lll}I_{x x} & I_{x y} & I_{x z} \\ I_{y x} & I_{y y} & I_{y z} \\ I_{z x} & I_{z y} & I_{z z}\end{array}\right)$.

In the body coordinate system, the angular motion equations of the aircraft under the action of external moment:

$$
\begin{aligned}
& M_{x}=p I_{x}-r I_{x}+q r\left(I_{z}-I_{y}\right)-p q I_{x} \\
& M_{y}=q I_{y}+p r\left(I_{x}-I_{z}\right)+\left(p^{2}-r^{2}\right) I_{x} \\
& M_{z}=r I_{x}-p I_{x}+p q\left(I_{y}-I_{x}\right)+q r I_{x}
\end{aligned}
$$

The torque equations are as follows:

$$
\begin{gathered}
p=\left(c_{1} r+c_{2} p\right) q+c_{3} M_{x}+c_{4} M_{z} \\
q=c_{s} p r-c_{6}\left(p^{2}-r^{2}\right)+c_{7} M_{y} \\
r=\left(c_{8} p-c_{2} r\right) q+c_{4} M_{x}+C_{9} M_{z} \\
c_{1}=\frac{\left(I_{y}-I_{z}\right) I_{z}-I_{x z}^{2}}{\Lambda}, c_{2}=\frac{\left(I_{x}-I+I_{z}\right) I_{x}}{\Lambda}, c_{3}=\frac{I_{z}}{\Lambda}, c_{4}=\frac{I_{x z}}{\Lambda}, c_{5}=\frac{I_{z}-I_{x}}{I_{y}}, \\
c_{6}=\frac{I_{x}}{I_{y}}, c_{7}=\frac{1}{I_{y}}, c_{8}=\frac{\left(I_{x}-I_{y}\right) I_{x}+I_{x}^{2}}{\Lambda}, c_{9}=\frac{I_{x}}{\Lambda}, \Lambda=I_{x} I_{z}-I_{x}^{2} .
\end{gathered}
$$

From the above equations, the aircraft attitude equations of motion and the aircraft azimuth equations of motion are derived:

$$
\begin{gathered}
x_{e}=u \cos \phi \cos \psi+v(\sin \phi \sin \theta \cos \psi-\cos \phi \sin \psi)+w(\cos \phi \sin \theta \cos \psi+\sin \phi \sin \psi) \\
y_{e}=u \cos \theta \sin \psi+v(\sin \phi \sin \theta \sin \psi+\cos \phi \cos \psi)+w(\cos \phi \sin \theta \sin \psi-\sin \phi \cos \psi) \\
h=u \sin \theta \sin \psi-v \sin \phi \cos \theta-w \cos \phi \cos \theta \\
\theta=q \cos \phi-r \sin \phi \\
\psi=(q \sin \phi+r \cos \phi) / \cos \theta \\
\varphi=p+\tan \theta(q \sin \phi+r \cos \phi)
\end{gathered}
$$

This series of equations together form a closed system of equations from which a set of state vectors for an aircraft can be determined: $\mathrm{x}=\left(\mathrm{u}, \mathrm{v}, \mathrm{w}, \phi, \theta, \psi, \mathrm{p}, \mathrm{q}, \mathrm{r}, \mathrm{x}_{\mathrm{e}}, \mathrm{y}_{\mathrm{e}}, \mathrm{h}\right)^{\mathrm{T}}$. If the relevant characteristic parameters of the aircraft are determined, the force and moment of the aircraft space can be determined according to the speed, attitude and position of the aircraft. Thus, applying the closed equations described above, the movement state of the aircraft at any time can be obtained. 


\section{The Level of CPS Physical Modeling}

In order to clearly model the aircraft system, the level of modeling of the CPS physical world is given. As shown in Figure 1, the specification layer clearly defines the objects of modeling, and relates to the system, system behavior, system environment boundary, and system. Different roles [4]. The physical system is divided into different components and describes their interactions. The equations and algorithms of the physical layer formal model components, the physical component equations are based on physical formula theorem, application rules from data sources, and interactive model standards [5]. The logical component algorithm is implemented in the control unit of the system. The mathematics layer collects all the physical component equations and derives a standard form of mathematics, such as an ordinary differential equation or a differential algebraic equation that is suitable for model simulation. Finally, at the runtime layer, the model is connected to simulation algorithms and performs model simulations.

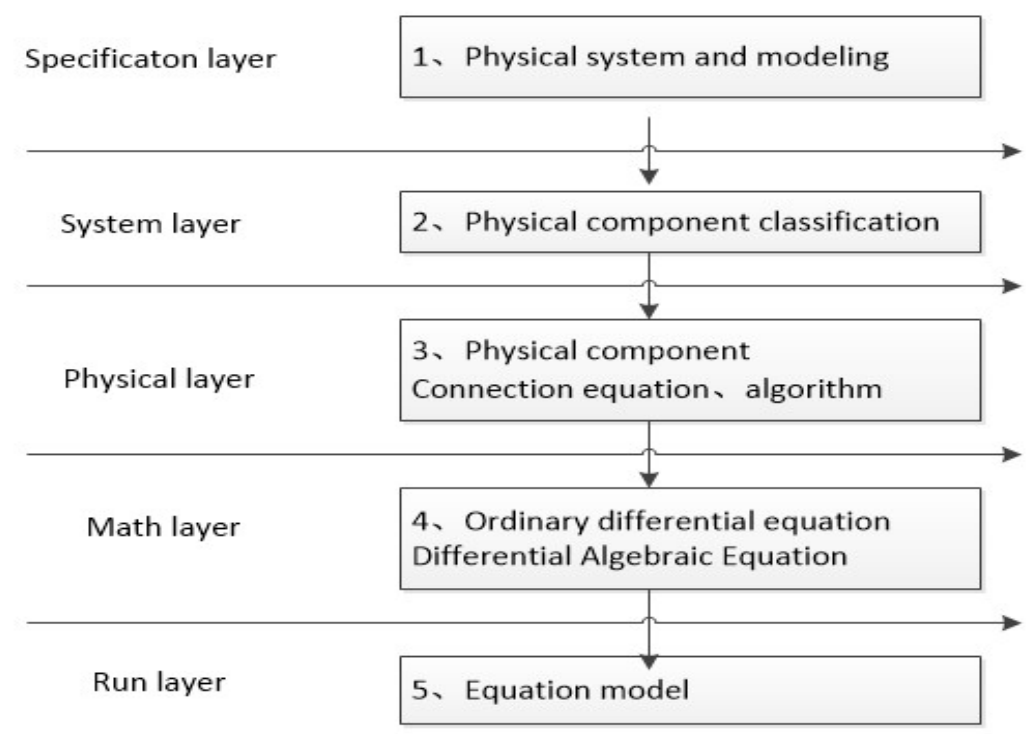

FIGURE 1. Physical model hierarchy

\section{The Description of Aircraft Software System}

Modern general-purpose transportation aircraft mainly include flight control systems, flight management systems, automatic flight systems, communication systems, navigation systems, and electronic instrument systems.

1) The flight control system is the general term for all the components and devices used to transmit maneuvering instructions on the aircraft to drive the rudder surface. It is used to control the attitude, speed and trajectory of the aircraft.

2) The flight management system is a computer system that integrates multiple onboard electronic systems [6]. It provides flight time, distance, speed, economic profile, and altitude predictions. Under the control of the FMS, the aircraft can achieve full-automatic navigation and can complete the entire flight process with the best flight path.

3) The automatic flight system is to reduce the driver's strength and energy, improve the accuracy of aircraft flight, and ensure the safety and quality of the aircraft to complete various tasks.

4) The communication system is mainly used to realize the mutual communication between the aircraft and the ground, between the aircraft and the aircraft, as well as to carry out inter-machine calls, passengers' broadcasting, recording of voice signals and providing audio and visual entertainment signals to passengers.

5) Navigation systems can be divided into inertial navigation, radio navigation and satellite navigation. Inertial navigation systems are used to achieve remote autonomous navigation. Radio navigation systems are used to guide aircraft to complete missions safely and efficiently along selected routes. Satellite navigation system can provide global, all-weather, high-precision, timely navigation and positioning services. 
6) Electronic instrumentation systems include atmospheric data meters, inertial meters, radio meters, electronic integrated meters, and warning systems. It is mainly used to display all kinds of data information and warning information so that the pilot can understand the flight status of the aircraft in real time.

\section{CONCLUSION}

The flight information physical fusion system is the application of CPS in the field of aviation. This article mainly introduces the characteristics of CPS and CPS and describes the physical information model of the aircraft. The aircraft's flight dynamics equation is given by establishing a ground coordinate system, a body coordinate system and a speed coordinate system. The equation can be used to solve the aircraft's movement at any time. Finally, the composition of the basic aircraft system was introduced. With the expansion of aviation scale, the flight information physics system will play an increasingly important role. By modeling the flight system, it is possible to analyze and optimize the flight schedule. It is of great help to the safety and efficiency of the flying business.

\section{ACKNOWLEDGMENTS}

This work is supported by the national natural science foundation of China under grant (No.61572142), natural science foundation of Guangdong province under grant (No.2015A030313490).

\section{REFERENCES}

1. Zhongjie Wang, Lulu Xie, Cyber-physical Systems: A Survey [j]. Acta Automatica

2. Sinica,2011,37(10):1157-1166.

3. Cyber-Physical System white paper,2017.3.

4. Junjie Tang. Research on Equation-based Modeling and Simulation of Cyber Physical Systems[D]. Huazhong University of Science and Technology,2013.

5. Lei Zhang. Flight Simulation Modeling and Software Development for Flight Simulator[D]. Harbin Institute of Technology,2009.

6. Yelun Xiao, Wanchun Chen. Static, kinematic and dynamic methods for relative attitude motion of aircraft. China Space Science and Technology,2003.10:10-15.

7. Weitao Miao, FangFang Xue, Liangliang Wang. Research on Design Method of General Aircraft Flight Management System. Civil Aviation International Forum,2017.4:228-233. 